\title{
Parent and medical professional willingness to enroll children in a hypothetical pediatric optic neuritis treatment trial
}

\author{
Amy T. Waldman 1,3 ${ }^{*}$, Michael J. Shumski ${ }^{1}$, Matthew Jerrehian ${ }^{2,3}$ and Grant T. Liu ${ }^{2,3,4}$ \\ 1 Division of Child Neurology, Children's Hospital of Philadelphia, Perelman School of Medicine at the University of Pennsylvania, Philadelphia, PA, USA \\ ${ }^{2}$ Neuro-ophthalmology service, Children's Hospital of Philadelphia, Perelman School of Medicine at the University of Pennsylvania, Philadelphia, PA, USA \\ ${ }^{3}$ Department of Neurology, Perelman School of Medicine at the University of Pennsylvania, Philadelphia, PA, USA \\ ${ }^{4}$ Department of Ophthalmology, Perelman School of Medicine at the University of Pennsylvania, Philadelphia, PA, USA
}

\section{Edited by:}

Hanspeter E. Killer, Kantonsspital

Aarau, Switzerland

\section{Reviewed by:}

Jane W. Chan, University of Nevada

School of Medicine, USA

Dan Milea, Glostrup Hospital,

Denmark

Prem Subramanian, Johns Hopkins University School of Medicine, USA Heather Moss, University of Illinois at Chicago, USA

Andrew Go Lee, The Methodist

Hospital, USA

*Correspondence:

Amy T. Waldman, Division of Neurology, Children's Hospital of Philadelphia, Clinical Translational Research Building, 10th Floor, Room 10012, 3501 Civic Center Boulevard,

Philadelphia, PA 19104, USA.

e-mail:waldman@email.chop.edu
The optic neuritis treatment trial (ONTT) and subsequent studies have had a tremendous impact on the treatment and prognosis of optic neuritis and multiple sclerosis in adults. The results of these studies have been extrapolated to children; however, pediatric data are sparse. Using the method of prospective preference assessment, the willingness of parents and medical professionals to enroll children in a hypothetical Pediatric ONTT was assessed using a mock consent form and questionnaire. A three-arm trial was proposed: (1) intravenous corticosteroids, (2) high-dose oral corticosteroids, and (3) an oral placebo. The forms were completed by 198 parents and 49 physicians. After reviewing the hypothetical scenario, trial design, risks and benefits, and alternatives to the study, $21 \%$ of parents would enroll their children in the trial whereas $98 \%$ of medical professionals would enroll their patients. With medical professional recommendation, $43 \%$ of parents would enroll their children. The manner in which this hypothetical trial was presented to parents, specifically with respect to the recommendation of their child's health care team, influenced a parent's willingness to participate.

Keywords: optic neuritis, children, clinical trial, prospective preference assessment

\section{INTRODUCTION}

Optic neuritis is an acute, inflammatory disease of the optic nerve and is often the initial manifestation of multiple sclerosis (MS) in adults and children (Duquette et al., 1987; Sindern et al., 1992; Boiko et al., 2002; Optic Neuritis Study Group, 2008a; Bonhomme et al., 2009). The clinical profile may include sudden vision loss, dyschromatopsia, pain with eye movements, and visual field defects (Optic Neuritis Study Group, 1991; Beck et al., 1992). Recovery often occurs over the course of several months; however, patients may have long-lasting symptoms of visual impairment (Sanders et al., 1986; Fleishman et al., 1987; Beck and Cleary, 1993). Even if visual acuity returns to baseline, abnormalities in color vision, visual field, and contrast sensitivity may persist (Optic Neuritis Study Group, 2008b).

In adults, the optic neuritis treatment trial (ONTT) has shown that the administration of intravenous corticosteroids hastens visual recovery, but does not affect the long-term visual or neurologic prognosis when compared to placebo (Optic Neuritis Study Group, 2008b). Despite insufficient evidence in the pediatric age group of long-term efficacy and the potential for adverse reactions even with short-term therapy, most pediatric ophthalmologists and neurologists still prescribe a short course of intravenous corticosteroids as treatment for acute optic neuritis in children, largely based on the results of the adult trial (Cakmakli et al., 2009). Since children were not included in the ONTT, the applicability of its conclusions to the pediatric patient population is unknown. Moreover, the extrapolation of adult data may be unsafe, and regulatory agencies are demanding additional research in pediatrics, including neurology, ophthalmology, and the use of immunomodulating drugs (Roberts et al., 2003; Ward and Kauffman, 2007).

While we believe that performing a clinical trial in children with optic neuritis would be the optimal means of resolving the problem of generalizing the adult study's results to this patient population, there are several hurdles to its success. As with any clinical trial, there are medical, ethical, and practical considerations that must be addressed. The ONTT was a three-arm study [intravenous (IV) methylprednisolone or oral prednisone vs. placebo], and the trial concluded that oral steroids should not be used in the treatment of optic neuritis due to the lack of efficacy in regards to visual outcome and increased risk of recurrent disease (Beck and Cleary, 1993; Optic Neuritis Study Group, 2008b). Given these results, we questioned whether medical professionals would be willing to enroll their pediatric patients in a trial with an oral treatment arm.

In addition, trial enrollment may be threatened by the use of a placebo arm (Caldwell et al., 2003). Intravenous 
methylprednisolone followed by oral prednisone hastened visual recovery within the first 6 months after presentation and decreased the short-term (2-year) risk of MS compared to placebo; however, steroids did not alter the long-term visual or neurologic prognosis (Beck et al., 1992; Optic Neuritis Study Group, 2008a,b). Nevertheless, we hypothesized that, when dealing with the loss of vision in a child, parents would want the treatment, not placebo, arm.

Considering these factors, we performed a prospective preference assessment. This method allows investigators to determine factors that may influence enrollment in a clinical trial prior to formal recruitment so that the study design could be modified as needed (Halpern, 2002). The primary objective of this study was to determine whether parents and medical professionals would give consent for their children and pediatric patients, respectively, to participate in a hypothetical study similar to the ONTT in adults. The secondary objective was to determine whether a three-arm trial deterred parents and medical professionals from agreeing to participate.

\section{MATERIALS AND METHODS}

A questionnaire was administered to parents at The Children's Hospital of Philadelphia (CHOP) by a research assistant. A convenience sample of parents waiting to be seen in the Ophthalmology and Neurology clinics and on the inpatient Neurology floor was approached, and the purpose of the study was explained. The research assistant was available for questions but did not read or review the questionnaire with the parent.

The questionnaire described a hypothetical situation in which a child is diagnosed with optic neuritis and asked to participate in a research study. The details of a randomized, masked, placebocontrolled clinical trial for optic neuritis were described in the format of a mock consent form. This discussion included details of the proposed treatment arms, a description of randomization and masking, the risks and benefits associated with participating, and a summary of the results from the ONTT in adults, as well as the family's alternatives to participating.

Following the presentation of this hypothetical scenario, parents were asked a series of questions to assess their willingness to enroll their child in such a clinical trial for optic neuritis. They were first asked about the specific trial design detailed in the presentation, which will be referred to as the "primary trial design," in which the treatment arms were described as (1) IV steroids for 3 days, followed by oral steroids for 14 days, (2) highdose oral steroids for 3 days followed by low-dose oral steroids for 14 days, and (3) oral placebo for 17 days. Details regarding the specific corticosteroid and dosing were not specified for the treatment arms. Subjects were asked whether or not they would participate in the hypothetical trial by selecting "yes" or "no," and then provided space to document the reason for their choice.

After this initial query, parents were also asked if they would participate in several alternative trial designs (i.e., IV corticosteroids vs. placebo, without an oral arm; or IV corticosteroids vs. oral steroids, without a placebo arm). All parents were required to answer "yes" or "no" to the primary trial design. Unlike the followup questions regarding alternative trial designs, "do not know" was not provided as an answer choice.
In addition, parents were asked three objective (true/false) questions to assess whether they understood the information that was presented to them regarding the design of the trial. Finally, parents were asked about any prior experience they had participating in clinical trials and about the past medical history of their child. Demographic or identifying information about the parent and his or her child was not collected. Space was provided for additional thoughts or concerns.

A similar questionnaire was distributed to adult and pediatric ophthalmologists and other eye care professionals at the Scheie Eye Institute's 136th Anniversary Meeting Scientific Program (May 21, 2010, Philadelphia, PA, USA) and to child neurologists at CHOP (collectively referred to as medical professionals). The questionnaire included a summary of the results from the ONTT and an abbreviated study protocol, similar to the one given to parents.

Analogous to the parent questionnaire, medical professionals were first asked if they would refer a pediatric patient $(<18$ years of age) to participate in the proposed clinical trial with the primary trial design; however, an additional answer choice was made available to identify those who did not see patients in a clinical practice. Similarly, medical professionals were then posed the same question for several alternative trial designs. Finally, medical professionals were asked about their personal experience conducting or participating in clinical trials.

Primarily, we were interested in the proportion of parents and medical professionals who would be willing to participate in the primary trial design; therefore, results of the questionnaire were summarized by standard descriptive measures. Given this primary objective, if subjects failed to respond to the primary trial design question, then all subsequent responses were excluded from further analysis, and the entire form was excluded from our study. The secondary objective was to identify particular aspects of the proposed trial which affected the decision to enroll a child for both parents and physicians. A subanalysis (using a chi-square test) was performed to determine if there was a difference in response rate among those parents whose children had chronic medical problems, a history of corticosteroid use, or clinical trial experience (each was examined independently, multivariable analyses were not performed). To assess the willingness to participate in alternative designs, univariate analyses were performed comparing the distribution of responses through 95\% confidence intervals. All analyses were performed using Stata 10.0.

The study was reviewed and approved by The CHOP Institutional Review Board. All participants verbally consented to participate in the survey; written consent was implied by completing the survey.

\section{RESULTS}

PARENTS

A total of 234 questionnaires were distributed to parents at $\mathrm{CHOP}$ (120 at Ophthalmology clinic, 106 at Neurology clinic, and 8 on the CHOP inpatient Neurology floor). Questionnaires were returned by 206 parents; however, the primary trial design question was only answered by 198 parents ( $85 \%$ response rate; Table 1); 8 partially completed questionnaires were excluded from the analysis for insufficient data. 
After reading the questionnaire, 41 (21\%) parents would enroll their child in the primary trial design, and 157 (79\%) would not participate (Table 2). Among those willing to participate, 16 parents $(39 \%)$ would enroll to "help his or her child" and another three parents $(7 \%)$ specifically commented that the benefits outweighed the risks. Altruism (i.e., wanting to help other children and advance our knowledge of the optimal treatment for optic neuritis in children) was another reason for enrolling, cited by 11 parents $(27 \%)$. The space to provide a reason for enrolling was left blank by nine parents (22\%). Finally, two parents (5\%) cited the additional testing and monitoring that children in the trial would receive as the reason to participate.

Of the 157 parents who declined enrollment, 34 parents $(22 \%)$ cited the risk/benefit ratio and an additional 27 (17\%) reported the medication side effects as the primary reason. Factors related to the child's health were named by 23 parents $(15 \%)$, including a few parents who thought their children were too young or that their current medical conditions would make them ineligible for the study. Others stated that they would only consider trials

Table 1 | Baseline characteristics.

\begin{tabular}{lccc}
\hline & $\begin{array}{l}\text { No. of forms } \\
\text { distributed }\end{array}$ & $\begin{array}{l}\text { No. of forms } \\
\text { completed }\end{array}$ & $\begin{array}{l}\text { Response } \\
\text { rate (\%) }\end{array}$ \\
\hline LOCATION WHERE PARENTS WERE APPROACHED & \\
Ophthalmology out-patient clinic & 120 & 104 & 87 \\
Neurology out-patient clinic & 106 & 89 & 84 \\
Neurology inpatient clinic & 8 & 5 & 63 \\
All locations & 234 & 198 & 85 \\
SPECIALTY OF SURVEYED PHYSICIAN & & \\
Ophthalmology & 50 & 30 & 60 \\
Neurology & 30 & 19 & 63 \\
All specialties & 80 & 49 & 61
\end{tabular}

for life-threatening conditions. There were 18 parents (11\%) who wanted to discuss the trial with their own physician or another specialist; therefore, they declined enrollment. The placebo arm was a factor for 16 parents (10\%). While five parents were opposed to their children receiving the placebo, 11 parents declined the study because of the favorable outcome of the placebo group. For the remaining parents, $14(9 \%)$ did not provide a reason for declining to participate, $11(7 \%)$ did not want their children to be randomized (and would prefer they or their doctor picked the treatment), 8 (5\%) required more research, and 6 (4\%) opposed the participation of their children in research.

When all parents were asked if they would enroll their children if their doctor recommended it, 85 (43\%) would participate, 45 (23\%) would not participate, $64(32 \%)$ were unsure, and $5(3 \%)$ did not answer the question (Table 2). Of the 157 parents who initially refused enrollment, 49 (31\%) responded that they would participate if their doctor recommended it, 42 (27\%) would not participate, 55 (35\%) were uncertain, and 11 (7\%) did not respond to the question.

Regarding the medical history of their child, 82 (41\%) parents responded that their child had a chronic medical condition. The condition was specified by 73 parents, with the most common being seizures/epilepsy (11), optic glioma/neurofibromatosis type 1 (9), brain tumor (4), and asthma (4). In addition, specific questions addressed whether the child had a demyelinating disease, and a small subset of parents reported a history of optic neuritis (2), MS (1), or acute disseminated encephalomyelitis (1). Of the 188 parents who answered, most parents responded that their children were naive to IV and oral forms of steroids, $162(86 \%)$ and 139 (74\%), respectively, and 139 out of 189 who responded (74\%) had never been asked to participate in a clinical trial.

Analysis of baseline characteristics did not identify any statistically significant associations with willingness to participate in the primary trial design: prior clinical trial experience $(p=0.07)$, child

Table 2 | Parental willingness to participate.

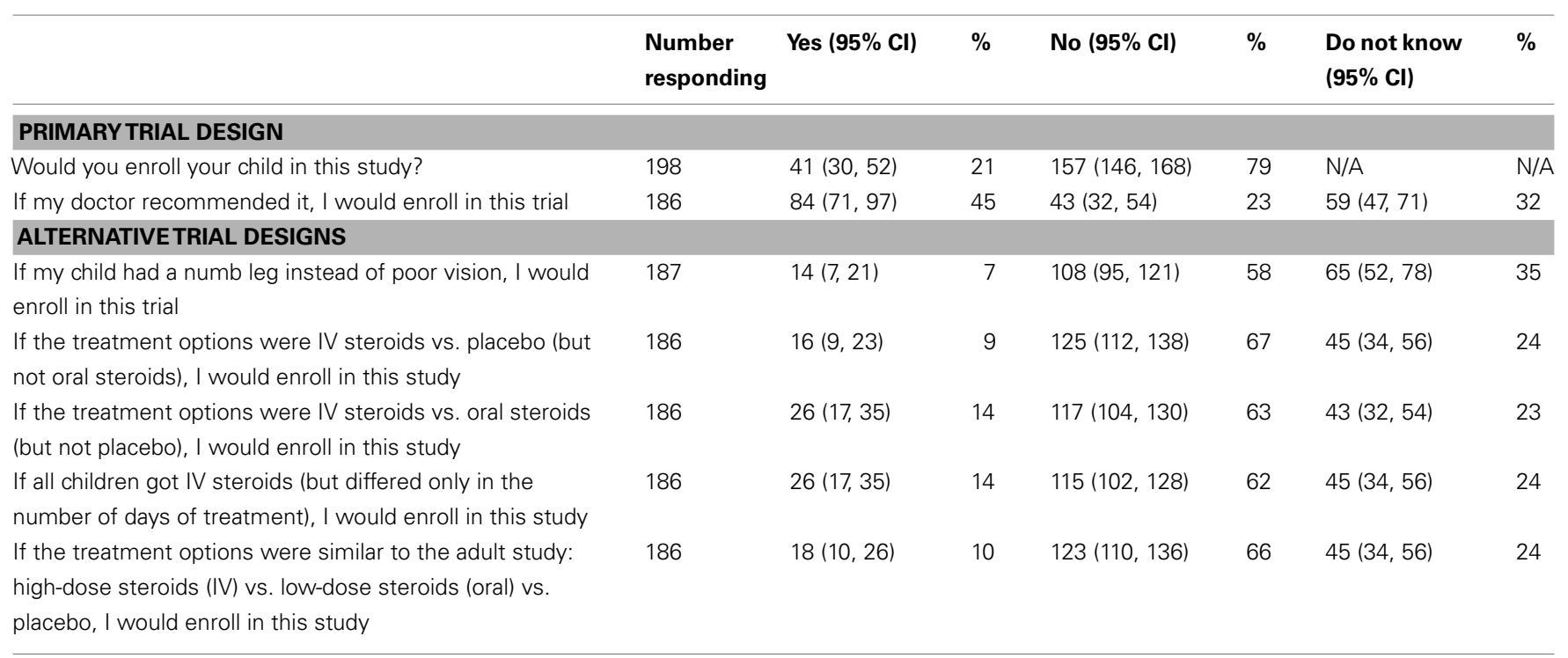

$N / A=$ not applicable; "Do not know" was not given as an answer choice. 
with chronic medical condition $(p=0.16)$, prior treatment with IV steroids $(p=0.12)$ or oral steroids $(p=0.15)$, or clinical setting (ophthalmology vs. neurology, $p=0.37$ ).

In response to three objective questions to determine if parents understood the clinical trial design, 188 responded to at least two questions. All three questions were answered correctly by 126 parents, and two out of three questions were answered correctly by an additional 22 parents (79\% combined, Table 3 ). Correctly answering all three objective questions was not associated with willingness to participate in the primary trial design $(p=0.49)$. Responses to a series of questions about alternative trial designs

Table 3 | Objective question responses.

\begin{tabular}{|c|c|c|c|c|}
\hline $\begin{array}{l}\text { True/false } \\
\text { questions }\end{array}$ & $\begin{array}{l}\text { Number } \\
\text { responding }\end{array}$ & True (\%) & False (\%) & $\begin{array}{l}\text { Do not } \\
\text { know (\%) }\end{array}$ \\
\hline $\begin{array}{l}\text { In a randomized } \\
\text { double-blind trial, } \\
\text { the physician } \\
\text { picks the } \\
\text { treatment that my } \\
\text { child receives }\end{array}$ & 188 & $20(11)$ & $151(80)$ & $17(9)$ \\
\hline $\begin{array}{l}\text { A placebo is a } \\
\text { sugar pill }\end{array}$ & 189 & $183(97)$ & $1(1)$ & $5(3)$ \\
\hline $\begin{array}{l}\text { If I enrolled in the } \\
\text { above trial } \\
\text { (primary trial } \\
\text { design), my child } \\
\text { would have a } 1 / 3 \\
\text { chance of being } \\
\text { on no medication }\end{array}$ & 184 & $143(78)$ & $21(11)$ & $20(11)$ \\
\hline
\end{tabular}

Bold/underline $=$ correct answer. are presented in Table 2. Overall, the willingness to participate decreased for all alternative study designs.

\section{MEDICAL PROFESSIONALS}

Questionnaires were distributed to 80 physicians and eye care professionals and 49 were returned at least partially completed $(61 \%$ response rate; Table 1). Over half of responding medical professionals $(57 \%)$ had experience conducting a clinical trial in some capacity. For the primary trial design, 43 medical and eye care professionals (98\%) would refer their pediatric patients for the trial, and $1(2 \%)$ declined (Table 4$)$. For each of the proposed alternative trial designs, 43 responses were received (Table 4). Similar to the parent questionnaire, the willingness to participate decreased for all alternative study designs.

\section{DISCUSSION}

In this study we administered a questionnaire to assess the willingness of parents and medical professionals to participate in a clinical trial for acute optic neuritis in the pediatric population. Results from a hypothetical trial confirming the willingness to participate in the study support the likelihood of participating in the actual trial (Halpern et al., 2001). While medical professionals were overwhelmingly supportive of a three-arm Pediatric ONTT, parents were less likely to give consent for the participation of their children. Compared to adult trials, recruitment for pediatric studies has proven to be more difficult (Caldwell et al., 2003).

Prospective preference assessment was an effective method to gather pretrial data that will influence its future design. First, the proportion of parents willing to participate in a trial is critical to calculate the sample size for such a trial to achieve optimal power. These results will be used to minimize under enrollment, which would threaten the trial's validity. Second, the willingness to participate did not increase when alternate study designs (for example, without a placebo or oral arm) were proposed. Third, the support

Table 4 | Medical professional willingness to participate.

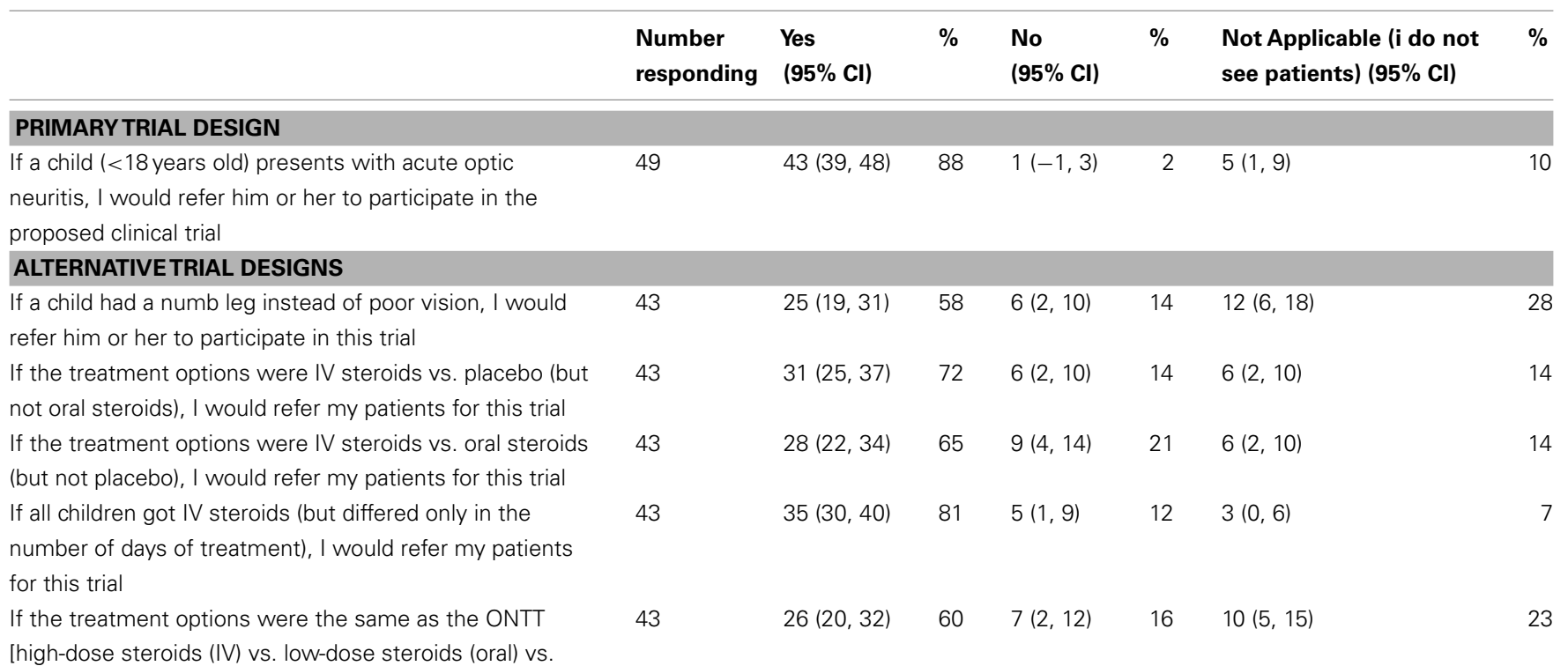

placebo], I would refer my patients for this trial 
of medical professionals is important to a parent in the decision to enroll.

While we hypothesized that the specifics of the design of this hypothetical study (i.e., a three-arm trial) would influence the decision to enroll, parental consent was determined by the same factors that affect any trial. The likelihood of enrolling in a trial is most often determined by the risk/benefit ratio (Tait et al., 2004). As a group, the risk/benefit ratio was the most common reason for parents' decision to enroll or decline participation in this study. Altruism is another common reason for participating in trials (Tait et al., 2003) whereas concern regarding the randomization and masking (blinding) processes are often deterrents for parents (Caldwell et al., 2003; Gross et al., 2006). These explanations were also commonly cited by parents in this study as the rationale for their decision.

Most parents correctly understood the implications of randomization, masking, and the use of a placebo, which were assessed using true/false questions to verify understanding of the concepts. Nevertheless, these factors present challenges in trial recruitment (Caldwell et al., 2003; Gross et al., 2006). While the use of a placebo arm is often a concern limiting trial enrollment, some families did not want their children exposed to corticosteroids and seemed to prefer no treatment. Additional trials were proposed to determine if enrollment would improve, for example, without a placebo or oral arm; however, parents and physicians were not more willing to participate in alternate study designs.

A trial assessing paresthesias, instead of vision loss, was posed to assess whether willingness to enroll would increase. This trial was proposed as the symptom may be considered more benign with decreased risk; however, neurologists commented that paresthesias may be due to spinal cord lesions; therefore, as a group, medical professionals were less likely to enroll their patients in such a trial. For the alternative questions, all participants were given the option of selecting "do not know" as an answer choice. This option was not given for the primary trial design since a definitive answer (yes or no) would be required for the actual trial. It is unclear how this option ("do not know") would have affected the outcome of the primary trial design.

The willingness to participate in a pediatric ONTT similar to the adult study doubled with a medical professional's recommendation. Previous studies have shown that approval from a physician impacts enrollment in clinical trials (Harth and Thong,

\section{REFERENCES}

Beck, R. W., and Cleary, P. A. (1993). Optic neuritis treatment trial. One-year follow-up results. Arch. Ophthalmol. 111, 773-775.

Beck, R. W., Cleary, P. A., Anderson, M. M. Jr., Keltner, J. L., Shults, W. T., Kaufman, D. I., Buckley, E. G., Corbett, J. J., Kupersmith, M. J., Miller, N. R., Savino, P. J., Guy, J. R., Trobe, J. D., McCrary, J. A., Smith, C. H., Chrousos, G. A., Thompson, H. S., Katz, B. J., Brodsky, M. C., Goodwin, J. A., Atwell, C. W., and the Optic Neuritis Study Group. (1992). A randomized, controlled trial of corticosteroids in the treatment of acute optic neuritis. N. Engl. J. Med. 326, 581-588.

Boiko, A., Vorobeychik, G., Paty, D., Devonshire, V., Sadovnick, D., and the University of British Columbia MS Clinic Neurologists. (2002). Early onset multiple sclerosis: a longitudinal study. Neurology 59, 1006-1010.

Bonhomme, G. R., Waldman, A. T., Balcer, L. J., Daniels, A. B., Tennekoon, G. I., Forman, S., Galetta, S. L., and Liu, G. T. (2009). Pediatric optic neuritis: brain MRI abnormalities

1995; Langley et al., 1998; Caldwell et al., 2003; Nabulsi et al., 2011). In addition to trust in the medical system, this increase in enrollment may be due to the shared responsibility for the child's health between the physician and parent (Tait et al., 2003). With a physician's consent, a parent may feel relieved of the burden of the decision to enroll in a clinical trial (Zupancic et al., 1997; Langley et al., 1998; Singhal et al., 2002; Shilling and Young, 2009). Physician recommendation is likely to enhance enrollment in a future pediatric ONTT; however, our motivation for asking this question was not to exploit the vulnerability of parents in the informed consent process. Instead, this underscores the importance of having the support of referring medical professionals when designing clinical trials, as these clinicians may impact a trial's success.

There are a number of limitations to this study. Demographic data was not collected. For the actual trial, recruitment would target all racial, ethnic, religious, and socioeconomic backgrounds; therefore, demographic data was not felt to provide additional insight regarding the willingness to enroll. Parents presenting to neurology and ophthalmology clinics in a single academic center were targeted; this convenience sample may not reflect the opinions of all parents. However, the institution is a tertiary care center which not only serves the community but also has a large referral base.

A mock consent form was used to assess the willingness to enroll as opposed to a structured interview with a research assistant. The latter has been shown to influence the willingness to enroll (Tait et al., 2003). Furthermore, the consent form is often intimating to parents and may alter their decision to participate after verbally agreeing to enroll (Pierro and Spitz, 1997). Since a research assistant and verbal explanation may increase the likelihood of enrolling, the use of a mock consent form rather than a research assistant may result in an underestimate of the willingness to enroll.

Using the method of prospective preference assessment, this study provided valuable insight into parent and medical professional interest in a hypothetical trial for children with optic neuritis. These results will improve the design of a future Pediatric ONTT.

\section{ACKNOWLEDGMENTS}

The authors wish to thank Scott Kasner, M.D., M.S.C.E., for his helpful hints and suggestions.

and risk of multiple sclerosis. $\mathrm{Neu}$ rology 72, 881-885.

Cakmakli, G., Kurne, A., Guven, A., Serdaroglu, A., Topaloglu, H., Teber, S., and Anlar, B. (2009). Childhood optic neuritis: the pediatric neurologist's perspective. Eur. J. Paediatr. Neurol. 13, 452-457.

Caldwell, P. H., Butow, P. N., and Craig, J. C. (2003). Parents' attitudes to children's participation in randomized controlled trials. J. Pediatr. 142, 554-559.

Duquette, P., Murray, T. J., Pleines, J., Ebers, G. C., Sadovnick, D., Weldon, P., Warren, S., Paty, D. W., Upton,
A., Hader, W., Nelson, R., Auty, A., Neufeld, B., and Meltzer, C. (1987). Multiple sclerosis in childhood: clinical profile in 125 patients. J. Pediatr. 111, 359-363.

Fleishman, J. A., Beck, R. W., Linares, O. A., and Klein, J. W. (1987). Deficits in visual function after resolution of optic neuritis. Ophthalmology 94, 1029-1035.

Gross, C. P., Krumholz, H. M., Van Wye, G., Emanuel, E. J., and Wendler, D. (2006). Does random treatment assignment cause harm to research participants? PLoS Med. 3, e188. doi:10.1371/journal.pmed.0030188 
Halpern, S. D. (2002). Prospective preference assessment: a method to enhance the ethics and efficiency of randomized controlled trials. Control. Clin. Trials 23, 274-288.

Halpern, S. D., Metzger, D. S., Berlin, J. A., and Ubel, P. A. (2001). Who will enroll? Predicting participation in a phase II AIDS vaccine trial. J. Acquir. Immune Defic. Syndr. 27, 281-288.

Harth, S. C., and Thong, Y. H. (1995). Parental perceptions and attitudes about informed consent in clinical research involving children. Soc. Sci. Med. 41, 1647-1651.

Langley, J. M., Halperin, S. A., Mills, E. L., and Eastwood, B. (1998). Parental willingness to enter a child in a controlled vaccine trial. Clin. Invest. Med. 21, 12-16.

Nabulsi, M., Khalil, Y., and Makhoul, J. (2011). Parental attitudes towards and perceptions of their children's participation in clinical research: a developing-country perspective. $J$. Med. Ethics 37, 420-423.

Optic Neuritis Study Group. (1991). The clinical profile of optic neuritis: experience of the Optic Neuritis Treatment Trial. Arch. Ophthalmol. 109, 1673-1678.

Optic Neuritis Study Group. (2008a). Multiple sclerosis risk after optic neuritis: final ONTT follow-up. Arch. Neurol. 65, 727-732.

Optic Neuritis Study Group. (2008b). Visual function 15 years after optic neuritis: a final follow-up report from the Optic Neuritis Treatment Trial. Ophthalmology 115, 1079-1082.

Pierro, A., and Spitz, L. (1997). Informed consent in clinical research: the crisis in paediatrics. Lancet 349, 1703.

Roberts, R., Rodriguez, W., Murphy, D., and Crescenzi, T. (2003). Pediatric drug labeling: improving the safety and efficacy of pediatric therapies. JAMA 290, 905-911.

Sanders, E. A., Volkers, A. C., van der Poel, J. C., and van Lith, G. H. (1986). Estimation of visual function after optic neuritis: a comparison of clinical tests. Br. J. Ophthalmol. 70, 918-924.

Shilling, V., and Young, B. (2009). How do parents experience being asked to enter a child in a randomised controlled trial? BMCMed. Ethics 10 1-11. doi:10.1186/1472-6939-10-1

Sindern, E., Haas, J., Stark, E., and Wurster, U. (1992). Early onset MS under the age of 16: clinical and paraclinical features. Acta Neurol. Scand. 86, 280-284.

Singhal, N., Oberle, K., Burgess, E. and Huber-Okrainec, J. (2002). Parents' perceptions of research with newborns. J. Perinatol. 22, 57-63.

Tait, A. R., Voepel-Lewis, T., and Malviya, S. (2003). Participation of children in clinical research: factors that influence a parent's decision to consent. Anesthesiology 99, 819-825.

Tait, A. R., Voepel-Lewis, T., and Malviya, S. (2004). Factors that influence parents' assessments of the risks and benefits of research involving their children. Pediatrics 113, 727-732.

Ward, R. M., and Kauffman, R. (2007). Future of pediatric therapeutics: reauthorization of BPCA and PREA. Clin. Pharmacol. Ther. 81, 477-479.

Zupancic, J. A., Gillie, P., Streiner, D. L., Watts, J. L., and Schmidt, B. (1997). Determinants of parental authorization for involvement of newborn infants in clinical trials. Pediatrics 99, e6.

Conflict of Interest Statement: The authors declare that the research was conducted in the absence of any commercial or financial relationships that could be construed as a potential conflict of interest.

Received: 12 October 2011; accepted: 13 November 2011; published online: 29 November 2011.

Citation: Waldman AT, Shumski MJ, Jerrehian M and Liu GT (2011) Parent and medical professional willingness to enroll children in a hypothetical pediatric optic neuritis treatment trial. Front. Neur. 2:75. doi: 10.3389/fneur.2011.00075

This article was submitted to Frontiers in Neuro-ophthalmology, a specialty of Frontiers in Neurology.

Copyright (c) 2011 Waldman, Shumski, Jerrehian and Liu. This is an open-access article distributed under the terms of the Creative Commons Attribution Non Commercial License, which permits noncommercial use, distribution, and reproduction in other forums, provided the original authors and source are credited. 